\title{
METODOLOGIA DE AQUISIÇÃO DA COMPONENTE RESISTIVA DA CORRENTE DE FUGA DE PARA-RAIOS DE ZNO UTILIZANDO TRANSFORMADA RÁPIDA DE FOURIER
}

\author{
Mellina M. Lisboa - mellina.lisboa@itec.ufpa.br* \\ Pedro Henrique de M. Barbosa - pedrombarbosa.eng@gmail.com* \\ Joao Henrique do R. Ribeiro - joaohenriquerosario@ gmail.com* \\ Stephany Carla C. Costa - stephany.carla17@gmail.com* \\ Marcus Vinicius A. Nunes - mvan@ufpa.br* \\ Universidade Federal do Pará* \\ Rua Augusto Corrêa, 01* \\ 66075-110 - Belém - Pará*
}

Resumo: Levando em consideração a relevância do para-raios para os sistemas elétricos de potência, faz-se necessário a inspeção do mesmo. Uma das principais formas de monitoração se dá pela medição da corrente que flui pelos terminais do para-raios, quando este se encontra em operação: a corrente de fuga. Essa corrente apresenta duas componentes: capacitiva e resistiva; esta última sofre influência tanto da tensão quanto da temperatura e, portanto, é um indicador das condições de operação do equipamento. O estudo dessa componente é de suma importância, haja vista que a análise do seu nível busca evitar complicações que o equipamento sofre em virtude das variações climáticos e perturbações referentes aos procedimentos por ele realizados e, dessa forma, evitar falhas e ampliar a vida útil do para raio. Devido a isso, o presente trabalho tem como finalidade apresentar uma metodologia de medição de corrente de fuga total, no LEAT-UFPA, e a aquisição da magnitude da componente resistiva. A obtenção foi realizada por meio de uma rotina computacional, a qual baseia-se na Transformada Rápida de Fourier (FFT).

Palavras-chave: Para raios. Corrente de Fuga. Transformada Rápida de Fourier.

\section{INTRODUÇÃO}

Os para-raios de Óxido de Zinco $(\mathrm{ZnO})$ são um dos equipamentos de proteção mais utilizados no Sistema Elétrico de Potência, protegendo-o de possíveis ocorrências de sobretensões. Eles atuam como limitadores de tensão, retendo a passagem de determinados valores preestabelecidos, as quais podem atingir os equipamentos a serem protegidos, como transformadores, reatores, banco de capacitores etc. (ÁLVARES, 2008).

Tendo em vista que o para-raios está sujeito às mais diversas situações, seja pelas intempéries ambientais (umidade e poluição do ar) ou os estresses resultantes das operações por ele efetuadas, torna-se imprescindível a inspeção no mesmo. Tal medida busca evitar falhas inesperadas, e assim, prolongar o limite de vida útil, além de garantir a sua substituição em casos mais urgentes.

Diante disso, uma das formas mais comuns e diretas de diagnosticar falhas no mesmo é por meio da medição e da análise da corrente de fuga, de modo que tal grandeza é formada por duas 
componentes: capacitiva e resistiva, e pode ser mensurada quando o para-raios sofre a aplicação de uma tensão senoidal, correspondente a $80 \%$ do valor da sua tensão nominal na região de baixa corrente. A componente resistiva é de mais comum a análise, pois a mesma sofre influência dos níveis de tensão e da temperatura no para-raios, além de ser responsável pelas perdas de energia nesse equipamento.

A corrente de fuga obtida ao submeter o para-raios ao sinal de tensão senoidal contém harmônicos, isso ocorre devido ao fato do equipamento possuir características não-lineares provenientes do $\mathrm{ZnO}$ (ARAUJO, 2010). Essa corrente, ao ser analisada computacionalmente, pode ter as suas componentes harmônicas identificadas, de modo que a terceira indica o nível da corrente resistiva. Para essa análise, pode-se utilizar as Séries e Transformadas de Fourier.

Afim de atender ao mercado de energia regional, à demanda de formação de mão-de-obra qualificada e à produção de conhecimento científico, Universidade Federal do Pará (UFPA) em parceria com a Financiadora de Estudos e Projetos (FINEP) inaugurou, em 2017, o Laboratório de Alta e Extra-Alta Tensão (LEAT). Ao contar com uma estrutura moderna, o laboratório é capaz de realizar ofertar diversos serviços e ensaio em equipamentos de baixa, média e alta tensão.

Logo, este artigo busca apresentar uma metodologia de medição de corrente de fuga total em para-raios de $\mathrm{ZnO}$, no LEAT, a fim de contribuir, como um guia de testes, para a realização de ensaios em para-raios de outras classes no referido laboratório. Além disso, mostrar a aquisição da componente resistiva dessa corrente através de uma rotina computacional utilizando Transformada de Fourier.

\section{REVISÃO BIBLIOGRÁFICA}

\subsection{Corrente de Fuga}

Em condições normais de operação, uma corrente flui pelos terminais do para-raios, na ordem de $\mathrm{mA}$ (mili Ampère) ou $\mu \mathrm{A}$ (micro Ampère), denominada corrente de fuga. Esta é composta por duas componentes: capacitiva e resistiva. A primeira é muito superior em relação à segunda, por isso, ela é tida como majoritariamente capacitiva (URSINE, 2013).

A componente capacitiva está relacionada às capacitâncias parasitas e à permissividade dos grãos de $\mathrm{ZnO}$; apresentando uma forma de onda predominantemente senoidal e $90^{\circ}$ de defasagem em relação ao sinal de tensão aplicado aos terminais do para-raios. Enquanto que a componente resistiva se mantém em fase com a tensão aplicada e possui forma de onda distorcida devido às características não-lineares da resistência variável, a qual varia com o aumento da tensão. Segundo a IEC 60099-5 (1999), a componente resistiva varia de 5\% a 20\% do valor de pico da corrente de fuga total.

A componente resistiva possui caráter dissipativo. Desse modo, ela é responsável pelo aumento da temperatura no para-raios por efeito Joule; e passível às variações da frequência e da temperatura dos componentes dos varistores (BARRADAS, 2014).

A Figura 1 mostra o momento em que a corrente resistiva é suficientemente alta a ponto de distorcer a forma de onda da corrente de fuga total. A elevação do valor da referida componente torna o para-raios sujeito a uma avalanche térmica, contribuindo para uma possível degradação do mesmo. 
Figura 1- Forma de onda da tensão e corrente de fuga do para-raios em operação

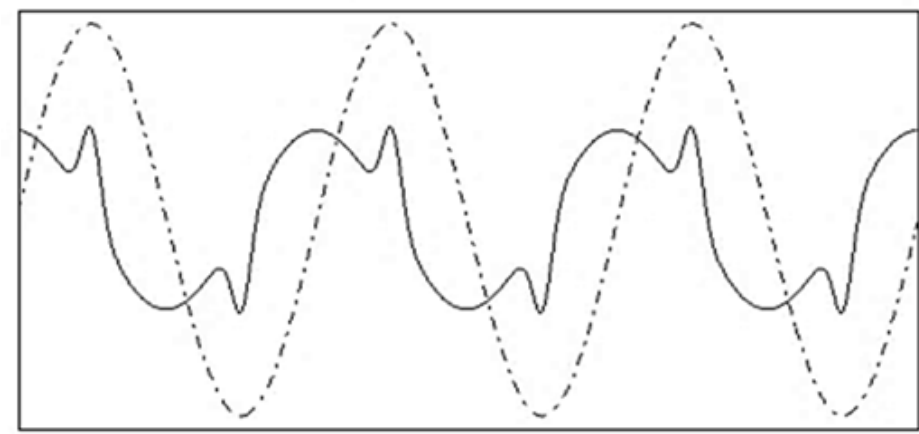

$$
\begin{array}{|l|}
\hline-- \text { Corrente de Fuga Total } \\
\hline- \text { Tensão }
\end{array}
$$

Fonte: URSINE, 2013

\subsubsection{Determinação indireta do componente resistiva por meio de análise harmônica da corrente de fuga}

Entre os métodos de aquisição indireta da componente resistiva da corrente de fuga, propostos pela IEC 60099-5 (1999), tem-se o B1. O referido método consiste no fato de que, ao sofrer a aplicação uma tensão senoidal, o para-raios gera uma corrente de fuga que contém conteúdo harmônico, das quais as componentes são provenientes da magnitude da componente resistiva e da curva característica do varistor. Isto é, o conteúdo harmônico é suscetível à variação da tensão e da temperatura do para-raios. De modo geral, a terceira componente é o indicador do nível da corrente resistiva.

\subsection{Séries e Transformada de Fourier}

A Série de Fourier é uma ferramenta largamente utilizada, visto que pode representar funções periódicas complexas e infinitas na forma de funções trigonométricas, simplificando assim, a manipulação e visualização destas funções. Além disso, elas podem representar tanto funções contínuas como não contínuas, o que torna este recurso ainda mais conveniente e eficiente (SANTOS,2015).

As aplicações são diversas e se estendem desde o significado físico, como os sinais elétricos, aos matemáticos como a resolução de equações diferenciais parciais. A forma geral da Série de Fourier é representada pela Equação (1), onde $\mathrm{a}_{0}, \mathrm{a}_{n}$ e $\mathrm{b}_{\mathrm{n}}$ são os termos numéricos que variam de acordo com a função a ser representada.

$$
f(t)=\frac{a_{0}}{2}+\sum_{n=1}^{\infty}\left[\text { an } \cos \left(\frac{n \pi t}{L}\right)+b n \cdot \operatorname{sen}\left(\frac{n \pi t}{L}\right)\right]
$$

Outra característica de Fourier é a FFT (Fast Fourier Transform ou Transformada Rápida de Fourier). Ela é usada, principalmente, em processamento de sinais, visto que realiza a decomposição espectral a partir de um sinal discreto e, dessa forma, torna possível a análise da amplitude das componentes de frequência do sinal (LIMA JÚNIOR,2014). A FFT pode ser demonstrada pela Equação (2).

$$
Y[k]=\sum_{l=1}^{N} y(l) \cdot w n^{(l-1) \cdot(k-1)}
$$


De modo que y é a função discreta, $Y[k]$ é a função transformada, $\mathrm{N}$ é o número de pontos da função e wn é descrita pela Equação (3).

$$
w n=e^{\frac{-2 \pi j}{N}}
$$

\subsection{LEAT-UFPA}

O Laboratório de Extra e Alta tensão (LEAT) foi inaugurado em junho de 2017 e está entre os cinco laboratórios neste ramo presentes no Brasil. O laboratório tem como principal objetivo a contribuição para a melhoria na qualidade dos serviços de transmissão e distribuição de energia, além de pesquisas de graduação e pós-graduação.

Fisicamente, o LEAT apresenta uma área total de $1153,7 \mathrm{~m}^{2}$ e é totalmente revestido por uma estrutura metálica, em razão das altas tensões geradas nos ensaios. Ademais, o laboratório trabalha diretamente com empresas dos setores de energia elétrica oferecendo diversos testes em equipamentos de alta tensão, atuando tanto em frequência industrial quanto com tensões e correntes impulsivas. Além dos ensaios de tensão residual e corrente de fuga em para raios, são ofertados ensaios de tensão suportável a impulso atmosférico à seco e sob a chuva, ensaio de tensão disruptiva a impulso atmosférico e entre outros. O laboratório faz uso de três grandes equipamentos: fonte série-ressonante, gerador de impulso de corrente e gerador de impulso de tensão

\section{METODOLOGIA}

É realizado o ensaio de medição de corrente de fuga total em um para-raios de classe 30 kV no Laboratório de Alta e Extra-Alta Tensão da Universidade Federal do Pará (LEATUFPA). Posteriormente, as curvas adquiridas no ensaio são carregadas na rotina computacional para adquirir a componente resistiva.

O procedimento, ilustrado na Figura 2, consiste no ensaio gerenciado pelo sistema de controle (1), o qual aciona o transformador de excitação (2) para que este carregue a fonte sérieressonante (3). Segue-se, então, a aplicação da tensão nos terminais do para-raios (4). O circuito resistivo (5) viabiliza a medição da corrente de fuga total; e o divisor de tensão capacitivo (6) mede a queda de tensão nos terminais do para-raios. Todos os dados medidos são captados pelo osciloscópio (7) e deste, é extraído um arquivo CSV e enviado à rotina computacional (8) para a aquisição do valor de pico da componente resistiva da corrente de fuga.

Figura 2 - Estrutura metodológica para aquisição da corrente de fuga resistiva

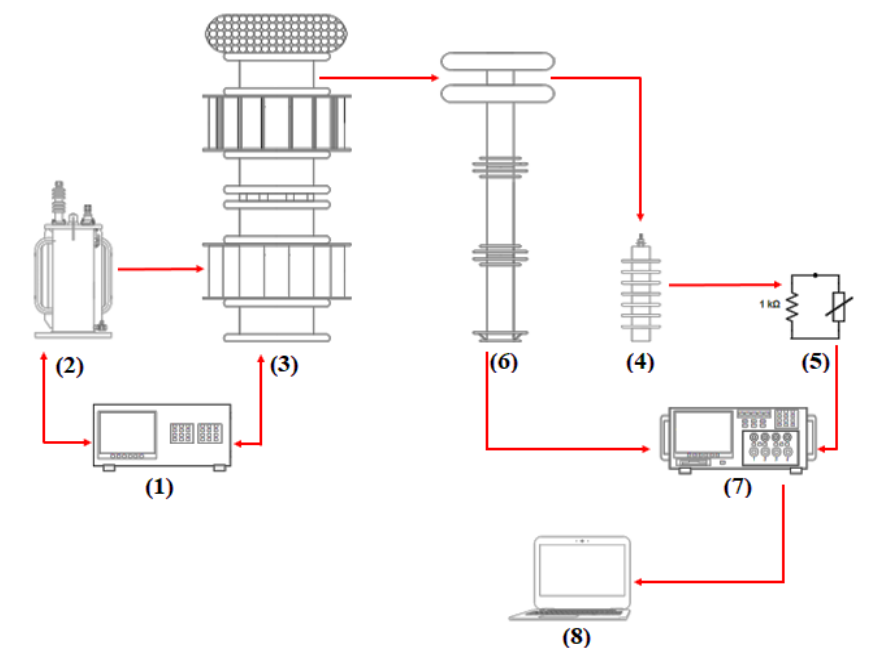




\subsection{Ensaio de Medição de Corrente de Fuga Total}

Para a obtenção da curva da corrente de fuga total, utilizou-se o arranjo da Figura 3. O circuito é carregado com a fonte série-ressonante e é medida a queda de tensão no divisor de tensão capacitivo e na resistência equivalente de $1 \mathrm{k} \Omega$ contida em uma caixa de acrílico.

Figura 3 - Arranjo de medição de corrente de fuga total

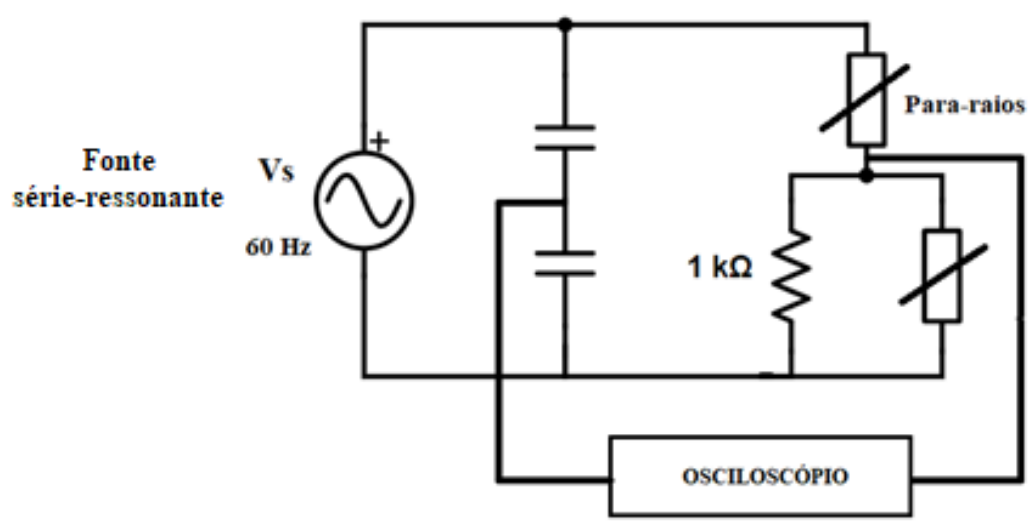

A resistência equivalente em questão é composta por uma associação série/paralelo de resistores de $1 \mathrm{k} \Omega$, a qual representa um circuito resistivo; o varistor em paralelo neste circuito é responsável por impedir que haja sobretensões no circuito, além de proteger o sistema de aquisição (osciloscópio).

As curvas de tensão são adquiridas por meio da utilização de um osciloscópio conectado ao circuito através de um conector BNC. Posteriormente, é calculada a corrente de fuga total mediante a manipulação algébrica da Lei de Ohm, apresentada na Equação (4), isto é, o quociente entre os valores de tensão obtida (U) e da resistência equivalente (Req) do divisor resistivo $(1 \mathrm{k} \Omega)$.

$$
I_{T}=\frac{U}{R_{e q}}
$$

\subsection{Aquisição da Componente Resistiva}

Baseado no método B1 de aquisição indireta da componente resistiva da corrente de fuga, a partir da posse dos valores de tensão no para-raios e da corrente de fuga total, foi possível calcular, através do algoritmo FFT contido no módulo em Python scipy.fftpack, o conteúdo harmônico presente na corrente de fuga total. Além disso, outros módulos importantes como xlrd e matplotlib foram utilizados, respectivamente, para capturar os dados no arquivo CSV e plotar os resultados. O fluxograma do algoritmo desenvolvido durante o trabalho é apresentado na Figura 4. 
Figura 4 - Algoritmo para determinação dos harmônicos presente na corrente de fuga total

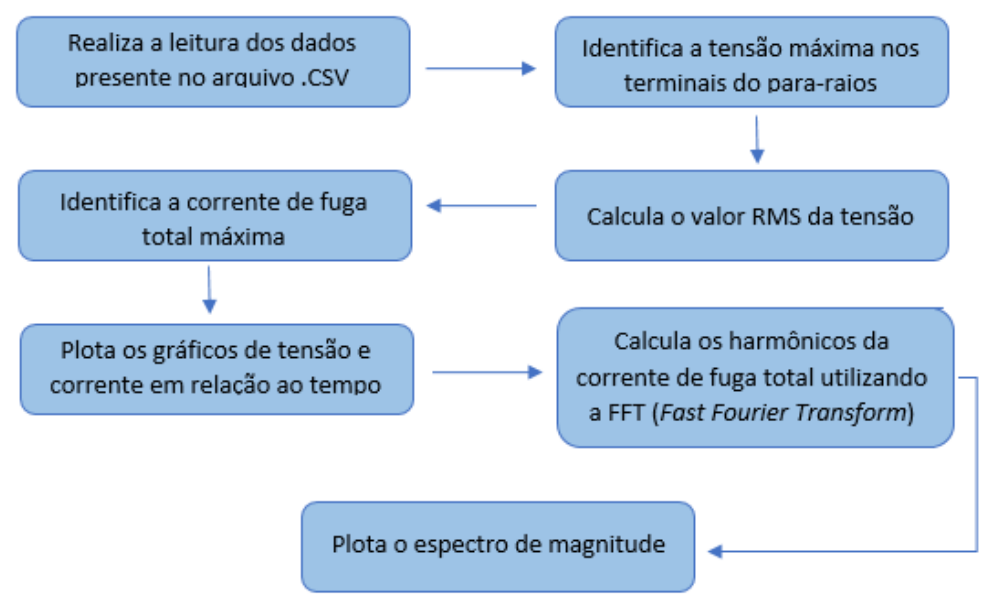

\section{RESULTADOS}

\subsection{Medição de Corrente de Fuga}

A NBR 16050 (ABNT, 2010) recomenda que os ensaios de medição de corrente de fuga em para-raios de $\mathrm{ZnO}$ sejam realizados por, no mínimo, 10 segundos e sob uma condição de temperatura contida entre o intervalo de $5{ }^{\circ} \mathrm{C}$ a $40{ }^{\circ} \mathrm{C}$. Na sala de testes do LEAT-UFPA, identificou-se que as condições do ambiente eram: $29,3^{\circ} \mathrm{C}$ e $80 \%$ de umidade.

Após montar a estrutura de do circuito de ensaio identificada na Figura 5, mediu-se os valores apresentados no Quadro 1, destacando que o pico da corrente de fuga total medida é de $0,432 \mathrm{~mA}$.

Figura 5 - Montagem do circuito de medição de corrente de fuga total

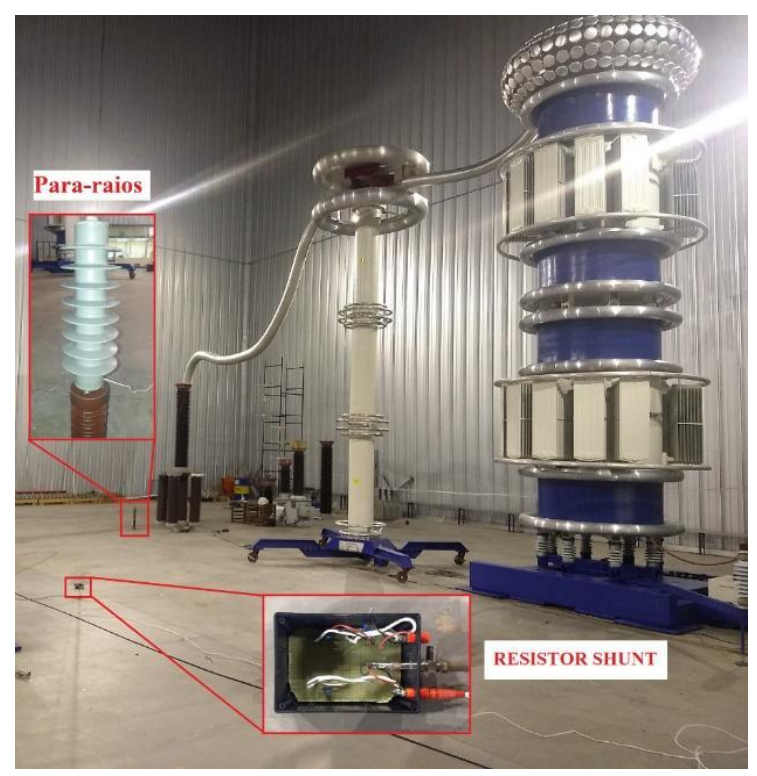


(C) COBENCE 2020

"Os desafios para formar hoje o engenheiro do amanhã"
$\mathrm{Ol} \mathrm{a} \mathrm{O3}$ de dezembro Evento On-line

Quadro 1 - Valores de tensão e corrente medidos

\begin{tabular}{|c|c|}
\hline Grandezas Medidas & Valores \\
\hline Tensão nos terminais do para-raios (rms) & $25,234 \mathrm{kV}$ \\
\hline Queda de tensão no circuito resistivo (pico) & $0,432 \mathrm{~V}$ \\
\hline Corrente de fuga total (pico) & $0,432 \mathrm{~mA}$ \\
\hline
\end{tabular}

\subsection{Aquisição da Componente Resistiva da Corrente de Fuga}

Com base no fluxograma da Figura 4, após o processamento dos dados é possível visualizar os resultados obtidos nas medições m laboratório, dentre eles: os gráficos de tensão nos terminais do para-raios e corrente de fuga total, representados pelas Figuras 6 e 7, respectivamente.

Figura 6 - Tensão nos terminais do para-raios em relação ao tempo

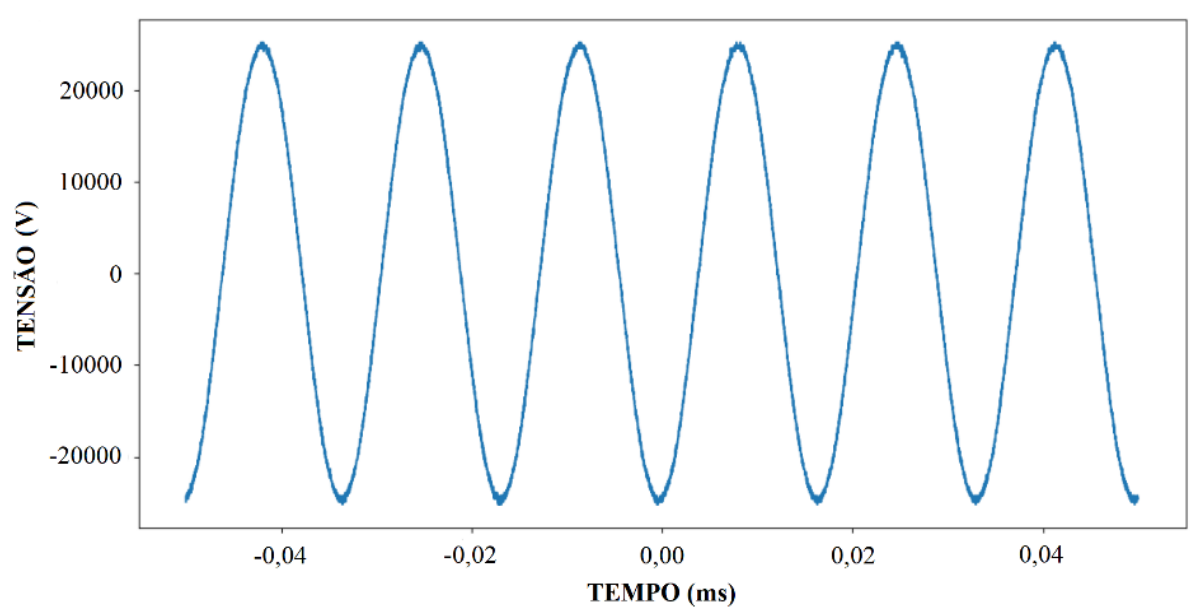

Figura 7 - Corrente de fuga total em relação ao tempo

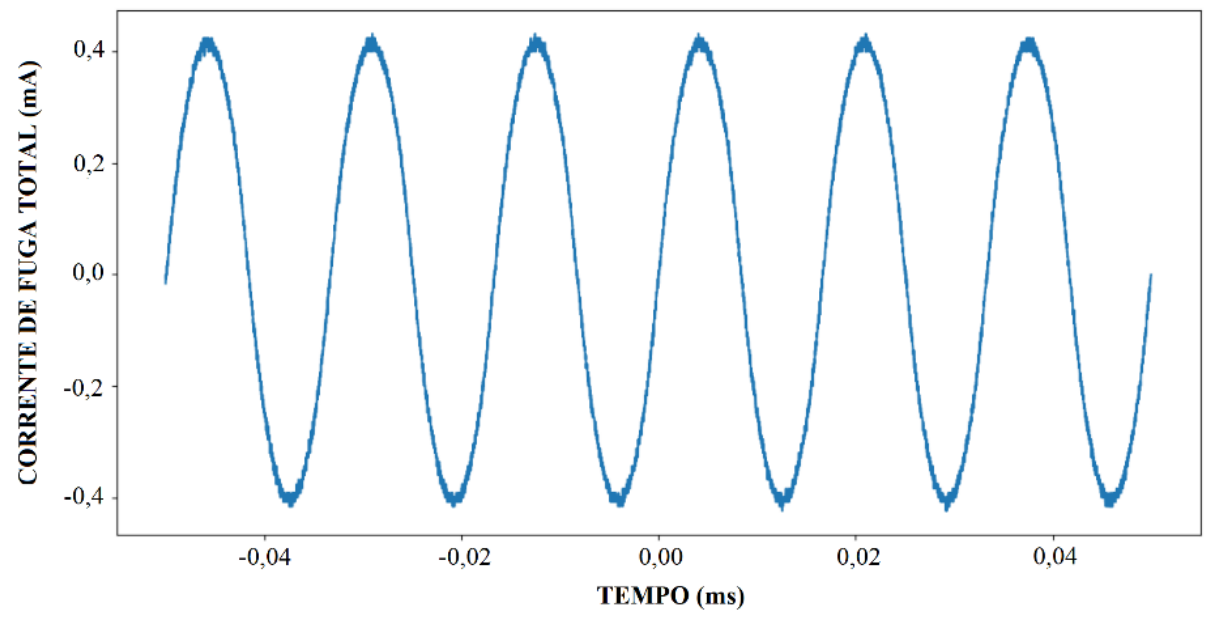

Ao submeter os dados da corrente de fuga total à FFT, foi possível adquirir o seu espectro de magnitude (Figura 8), da qual pode-se observar os valores de algumas componentes harmônicas impares. A primeira componente $(60 \mathrm{kHz})$, a terceira $(180 \mathrm{kHz})$ e a quinta $(300$ $\mathrm{kHz}$ ) são iguais a, respectivamente, 0,422 mA, 0,015 mA e 0,004 mA. 
Figura 8 - Espectro de magnitude da corrente de fuga total

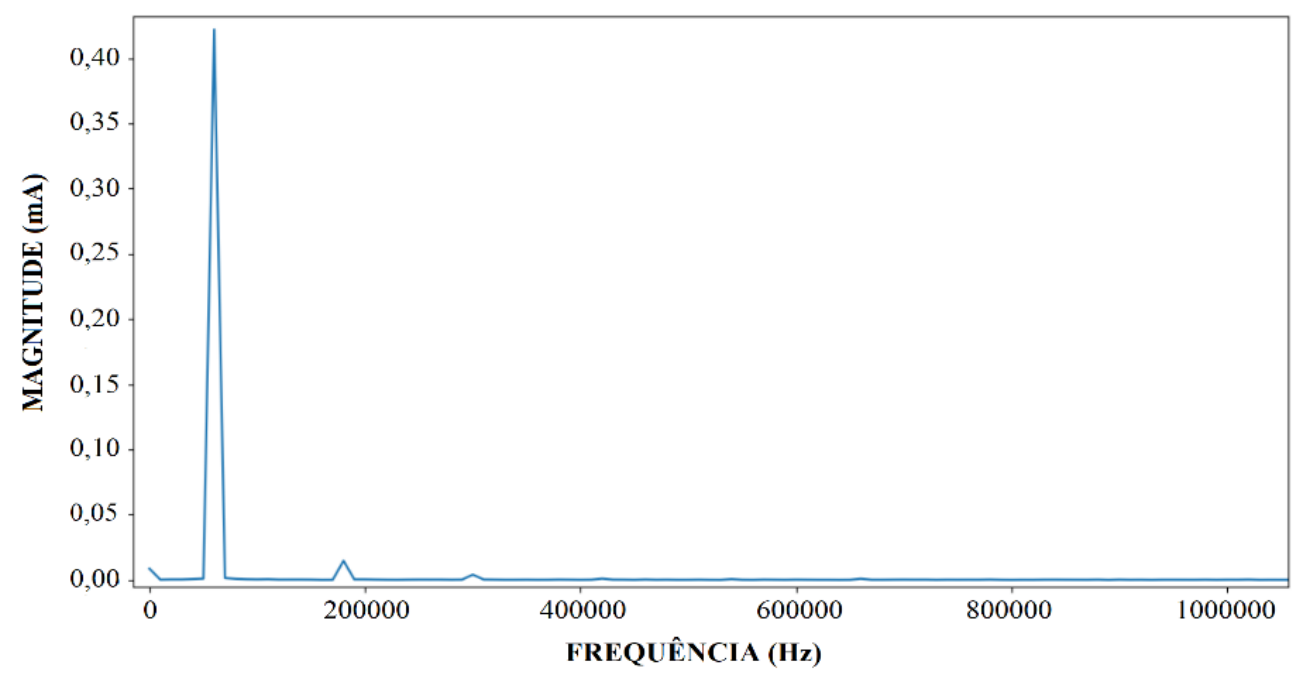

Assumindo que a terceira harmônica, representada pela Figura 9, indica o nível da componente resistiva do para-raios ensaio, pode-se inferir que essa última é igual a 0,015 mA, o que equivale à $3,47 \%$ da corrente de fuga total.

Figura 9 - Amplitude e frequência da terceira harmônica

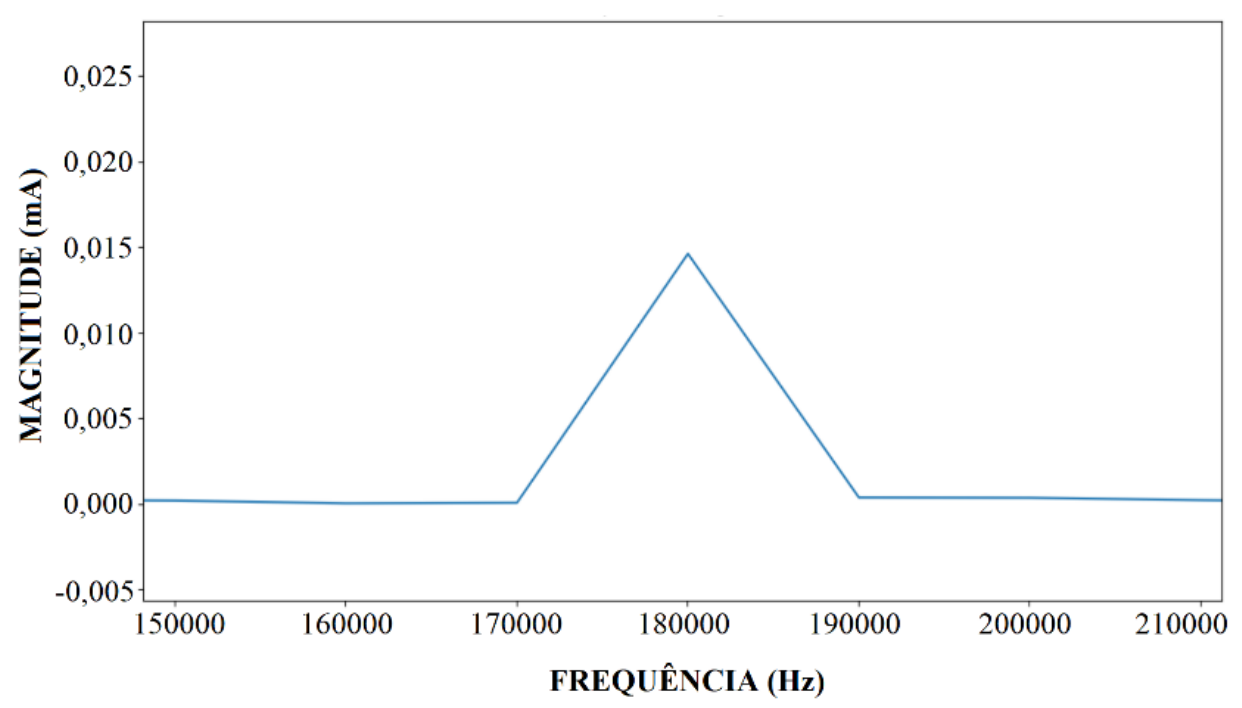

\section{CONSIDERAÇÕES FINAIS}

É inegável a importância da utilização do para-raios no sistema elétrico, dado que o mesmo exerce a função de proteção ao atuar como supressor de surtos e garantir a integridade dos demais equipamentos a serem protegidos. Mas assumindo que esses supressores estão sujeitos às mais diversas intempéries ambientais e físicas, recomenda-se a inspeção periódica nos mesmos a fim de se diagnosticar possíveis falhas. As práticas mais comuns baseiam-se na análise da corrente de fuga total, especialmente na sua componente resistiva.

Nesse contexto, o presente trabalho mostrou uma metodologia de medição experimental de corrente de fuga total e aquisição do nível da componente resistiva por meio de um software computacional desenvolvido em linguagem Python. 
A metodologia experimental do estudo consistiu no ensaio de medição de corrente de fuga total realizada no LEAT-UFPA, utilizando a fonte série-ressonante como circuito de entrada. Já a computacional, foi realizada pela decomposição da mesma corrente por meio da Transformada Rápida de Fourier.

A arquitetura do presente trabalho cumpriu os objetivos propostos quanto à medição e à decomposição da corrente de fuga em para-raios de $\mathrm{ZnO}$. Os dados adquiridos garantem a validação da rotina computacional desenvolvida e a aplicabilidade da Transformada Rápida de Fourier para a aquisição da componente resistiva, da mencionada corrente, de um para-raios. Além disso, a metodologia proposta poderá ser usada como guia de ensaios de corrente de fuga em para-raios de $\mathrm{ZnO}$ de outras classes de tensão, especialmente no LEAT.

\section{REFERÊNCIAS}

ARAUJO, Paulo Fernando de Matos. Metodologia e programa para decomposição da corrente de fuga de para-raios de Óxido de Zinco. (Monografia). Universidade Federal do Rio de Janeiro, Rio de Janeiro, 2010.

ASSOCIAÇÃO BRASILEIRA DE NORMAS E TÉCNICAS. NBR 16050: Para-raios de resistor não linear de óxido metálico sem centelhadores, para circuitos de potência de corrente alternada. Rio de Janeiro, 2010.

ÁLVARES, Ricardo Costa. Diagnóstico de falhas em para-raios utilizando termografia. Dissertação (Mestrado). Universidade Federal de Minas Gerais, Belo Horizonte, 2008.

BARRADAS, Raphael Pablo de Souza. Para-raios de Óxido de Zinco: Teoria e ensaios. Estudo de caso: ensaios de recebimento em para-raios de $12 \mathrm{kV}$. (Monografia) - Universidade Federal do Pará, Belém, 2014

INTERNATIONAL ELECTROTECHNICAL COMMISSION. IEC 60099-4: Surge arresters - Part 4: Metal-oxide surge arresters without gaps for a.c. systems. 1999

LIMA JÚNIOR, Geraldo Bezerra. Monitoramento de Para-Raios de Óxido de Zinco em Campo. Dissertação (Mestrado), Universidade Federal de Campina Grande, Paraíba, 2014.

SANTOS, Daniel Moreira dos. Estudo do THD da corrente de fuga de para raios como parâmetro indicador de degradação. Dissertação (Mestrado). Universidade Federal do Rio de Janeiro, Rio de Janeiro, 2015.

URSINE, Wagner Antônio Moreira. Monitoramento autônomo de para-raios de ZnO. (Dissertação de Mestrado). Universidade Federal de Minas Gerais, Belo Horizonte, 2013.

\section{METHODOLOGY OF ACQUISITION OF THE RESISTIVE COMPONENT OF THE LEAKAGE CURRENT OF ZNO SURGE ARRESTER USING FAST FOURIER TRANSFORM}

Abstract: Taking into account the relevance of the surge arrester for power systems, it is necessary to inspect it. One of the main forms of monitoring it is by measurement of the current flowing through the terminals of the surge arrester, when it is in operation: the leakage current. This current has two components: capacitive and resistive. The last one is influenced by both voltage and temperature and, therefore, is an indicator of operating conditions of the equipment. Such study is of paramount importance, since the analysis of the level of the same 
seeks to avoid complications that the equipment suffers due to climatic variations and disturbances related to the procedures performed by it and, thus, to avoid failures and extend the useful life of the surger arrester. Due to this, this paper aims to present a methodology for measuring total leakage current, in LEAT-UFPA, and the acquisition of the level of the resistive component. This was achieved by means of a computational routine, which is based on the Fast Fourier Transform (FFT).

Keywords: Surge arrester, Leakage Current, Fast Fourier Transform 